\title{
Exercise with Blood Flow Restriction to Improve Quadriceps Function Long After ACL Reconstruction
}

\author{
Authors \\ Affiliations \\ 1 Kinesiology and Integrative Physiology, Michigan \\ Technological University, Houghton, United States \\ 2 School of Health and Human Performance, Northern \\ Michigan University, Marquette, United States \\ 3 Physical Therapy, Aspirus Keweenaw Hospital, Laurium, \\ United States
}

Matthew A. Kilgas ${ }^{1,2}$, Lydia L.M. Lytle ${ }^{1,3}$, Scott N. Drum², Steven J. Elmer ${ }^{1}$

Key words

anterior cruciate ligament injury, quadriceps weakness, limb asymmetry, BFR exercise

accepted $\quad 17.06 .2019$

\author{
Bibliography \\ DOI https://doi.org/10.1055/a-0961-1434 \\ Published online: 23.7.2019 \\ Int J Sports Med 2019; 40: 650-656 \\ (c) Georg Thieme Verlag KG Stuttgart · New York \\ ISSN 0172-4622 \\ Correspondence \\ Prof. Steven J Elmer, PhD \\ Kinesiology and Integrative Physiology \\ Michigan Technological University \\ 1400 Townsed Dr \\ 49931 Houghton \\ United States \\ Tel.: + 1/906/487 2324, Fax: + 1/906/487 0985 \\ sjelmer@mtu.edu
}

\section{ABSTRACT}

Quadriceps atrophy and weakness can persist for years after anterior cruciate ligament reconstruction (ACLR). We evaluated the effectiveness of a home-based blood flow restriction (BFR) exercise program to increase quadriceps size and strength several years after ACLR. Nine adults with ACLR $(5 \pm 2$ yrs postsurgery, $\leq 90 \%$ symmetry in quadriceps size and strength) and nine uninjured controls volunteered. ACLR participants exercised at home for $25 \mathrm{~min}, 5 \times / \mathrm{wk}$ for 4 wks (single-leg knee extension, bodyweight half-squats, walking). Blood flow in only the involved leg was restricted using a thigh cuff inflated to $50 \%$ of limb occlusion pressure. Rectus femoris and vastus lateralis thickness and knee extensor strength were measured before and after training. Baseline and post-training symmetry (involved leg/uninvolved leg) indices were compared to uninjured controls. Rectus femoris and vastus lateralis thickness and knee extensor strength in the involved leg increased by $11 \pm 5 \%$, $10 \pm 6 \%$, and $20 \pm 14 \%$, respectively (all $P<0.01$ ). Compared to baseline, post-training knee extensor strength symmetry increased from $88 \pm 4$ to $99 \pm 5 \%(P<0.01)$ and did not differ from uninjured controls $(99 \pm 5 \%, P=0.95)$. Implementation of $B F R$ exercise at home was feasible, safe and effective. Results extend upon early post-operative application of BFR exercise for ACLR recovery and demonstrate that BFR can improve quadriceps function long after ACLR.

\section{Introduction}

After anterior cruciate ligament reconstruction (ACLR), many individuals do not completely regain their quadriceps size [1-3] and strength $[1,4-6]$. Persistent muscle and strength loss give rise to quadriceps asymmetry, which is associated with altered joint loading and gait mechanics [7,8], limited physical function [9], and increased risk for reinjury [10]. Moreover, chronic quadriceps asymmetry can lead to early onset of osteoarthritis [11,12]. While many investigators [13-15] have identified the presence of quadriceps asymmetry years after ACLR, few have focused on strategies to address these persistent impairments. Developing effective interventions to restore quadriceps function long after ACLR is not only warranted but is critical so individuals living with ACLR can maintain active lives.
Performing resistance exercise at $60-80 \%$ of one repetition maximum (1RM), $2-3 \times / w k$, for 8 wks is sufficient to increase quadriceps size and strength [16]. This exercise mode, however, is not always possible because higher loads are often contraindicated after ACLR. Exercise with blood flow restriction (BFR) is an effective method to build muscle and improve strength in clinical populations with knee pathologies $[5,17,18]$. For this exercise, an inflatable cuff applies compression to the limb to partially occlude blood flow while much lower loads are used (e. g. 20-30\% of 1RM). Takarada and colleagues [19] applied BFR days after ACLR surgery to minimize quadriceps muscle loss. In related work, other groups demonstrated that BFR exercise increased quadriceps muscle size and strength to a greater extent than traditional rehabilitative care 
following ACLR [20] and knee arthroscopy [21]. To date, there are no reports of using BFR exercise several years after ACLR.

Following discharge from supervised rehabilitation, patients are encouraged to continue with a home-based program. Continued adherence to this program is crucial, as patients are often permitted to return to limited sport activities with $85-90 \%$ quadriceps and/or limb symmetry $[8,9,22,23]$ with the expectation that full symmetry will be achieved over time. However, limited access to exercise equipment, time constraints and costs associated with follow-up treatment can pose barriers. Implementation of BFR exercise at home may circumvent some of these limitations as it can be performed with minimal equipment, facilitates strength gains quickly, and is inexpensive. Successful home-based programs also depend on patient motivation, amount of program education received, and previous experience exercising independently [24]. Thus, the implementation of a home-based BFR exercise program years after ACLR warrants further investigation before it could be used to aid with restoration of quadriceps size and strength.

Our purpose was to evaluate the effectiveness of a 4-wk, homebased BFR exercise program to increase quadriceps size, strength and symmetry several years after ACLR. We envisioned that a home program consisting of bodyweight and walking exercises with BFR would be feasible, safe and inexpensive. We hypothesized that BFR exercise would stimulate greater improvements in rectus femoris and vastus lateralis thickness and knee extensor strength in the involved leg compared to the uninvolved leg (i. e. non-BFR leg). We also hypothesized that symmetry in quadriceps size and strength would increase thus resulting in symmetry levels that would be closer to those for uninjured controls.

\section{Materials and Methods}

\section{Participants}

Nine adults who had previously undergone an ACLR and nine healthy uninjured controls volunteered to participate in this study (demographics reported in $>$ Table 1). Participants were between $18-44$ yrs of age and were physically active as they exercised at

- Table 1 Participant demographics.

\begin{tabular}{|c|c|c|c|}
\hline Variable & $\operatorname{ACLR}(n=9)$ & $\begin{array}{l}\text { Uninjured } \\
\text { Control }(n=9)\end{array}$ & $P$ value \\
\hline Age (yrs) & $26 \pm 8$ & $26 \pm 6$ & 0.81 \\
\hline Height (m) & $1.74 \pm 0.14$ & $1.73 \pm 0.08$ & 0.88 \\
\hline Body mass (kg) & $73 \pm 16$ & $71 \pm 12$ & 0.70 \\
\hline BMI $\left(\mathrm{kg} \cdot \mathrm{m}^{-2}\right)$ & $24 \pm 2$ & $24 \pm 3$ & 0.67 \\
\hline Body fat (\%) & $29 \pm 7$ & $27 \pm 5$ & 0.61 \\
\hline \multicolumn{4}{|l|}{ Sex } \\
\hline Male & 3 & 3 & \\
\hline Female & 6 & 6 & \\
\hline Time from ACLR (yrs) & $5 \pm 2$ & - & \\
\hline \multicolumn{4}{|l|}{ Graft type } \\
\hline Hamstring tendon & 3 & - & \\
\hline Patellar tendon & 6 & - & \\
\hline $\begin{array}{l}\text { Values are reported as } \\
\text { on } n=7 \text { for the } A C L R \text { gr }\end{array}$ & $\begin{array}{l}7 \pm \text { SD. Note the } \\
\text { and } n=8 \text { uninj }\end{array}$ & $\begin{array}{l}\text { body fat data are } \\
\text { ed control group. }\end{array}$ & orted \\
\hline
\end{tabular}

least $30 \mathrm{~min} 3-5 \times / \mathrm{wk}$ [16]. Individuals in the ACLR group were eligible to participate, if they: 1) had a unilateral ACLR > 2 yrs ago, 2) completed a post-operative rehabilitation program, 3) were cleared to return to physical activity by their orthopedic surgeon, and 4) exhibited $>10 \%$ difference in rectus femoris thickness, vastus lateralis thickness, or knee extensor strength between their involved and uninvolved leg (i. e. $<90 \%$ symmetry). Prior to the study, a physical therapist performed a clinical evaluation to verify that participants in the ACLR group were able to perform the baseline testing and home-based BFR exercise program. Specifically, these participants had to display negative signs and symptoms for knee ligamentous laxity, meniscal involvement, and patellar dysfunction. Participants also needed to demonstrate adequate muscular stability through the involved leg during a single leg squat, bilateral vertical jump, and forward, backward, and lateral lunge matrix. Individuals in the uninjured control group were eligible if they had no prior history of lower-extremity joint surgeries. This study was conducted in accordance with ethical standards in sport and exercise science research [25]. Experimental procedures were approved by our Institutional Review Boards and written informed consent was obtained.

\section{Experimental overview}

Baseline measures of rectus femoris and vastus lateralis thickness and single-leg knee extensor strength were assessed in the ACLR and uninjured control groups. Participants in the ACLR group performed BFR exercise at home $5 \times / w k$ for $4 w k s$. After the exercise program, muscle thickness and strength were assessed again. Baseline and post-training symmetry indices for ACLR participants were compared to baseline values for uninjured controls.

\section{Home-based BFR exercise}

Participants in the ACLR group visited the laboratory to complete a familiarization session where they were introduced to the BFR exercises. Each training session performed at home consisted of three exercises (single-leg knee extension, bodyweight half-squats, walking) and took $\sim 25 \mathrm{~min}$ ( $\triangleright$ Fig. 1). First, participants performed $3 \times 30$ single-leg knee extensions using their involved leg only with a resistance band. Next, participants performed $3 \times 30$ double-leg bodyweight half squats. Finally, participants performed $3 \times 2-$ min walking intervals at preferred walking speed. Rest was provided between sets ( $1 \mathrm{~min}$ ) and exercises ( $2 \mathrm{~min}$ ).

During each exercise, only blood flow in the involved leg was restricted using an 18-cm wide aneroid sphygmomanometer (Briggs, Healthcare, Waukegan, IL, USA). The cuff was wrapped around the thigh and inflated to $50 \%$ of the pressure required to occlude blood flow in the femoral artery. Limb occlusion pressure was determined in a seated position using Doppler Ultrasound as previously described $[26,27]$. The cuff remained inflated during the 1 -min rest between sets but was deflated during the 2 -min rest between exercises. During the 1 -min rest period between sets, participants returned to a seated position to check the pressure and adjust it if needed. Blood flow was partially occluded for $\sim 18$ of the 25 -min session. Each week participants performed one supervised training session in the laboratory to ensure proper movement form, adjust the resistance level, if needed, and verify that cuff pressure was correctly set. Finally, before each session participants performed a 
bodyweight half-squat to assess perceived muscle soreness in their legs using a visual analog scale $(0 \mathrm{~cm}$ - no soreness, $10 \mathrm{~cm}$ - very severe soreness) [28].

\section{Quadriceps size and strength}

Thickness of the rectus femoris and vastus lateralis were measured separately in the involved and uninvolved legs (ACLR group) and non-dominant and dominant legs (uninjured control group) using B-mode ultrasound (Logiq e BT12, GE Healthcare, Chicago, IL, USA). Participants were positioned supine $\left(10^{\circ} \mathrm{knee}\right.$ angle) while images of the rectus femoris and vastus lateralis were taken at $66 \%$ of the distance from the anterior superior iliac spine to the proximal patella. Muscle thickness was measured as the distance between

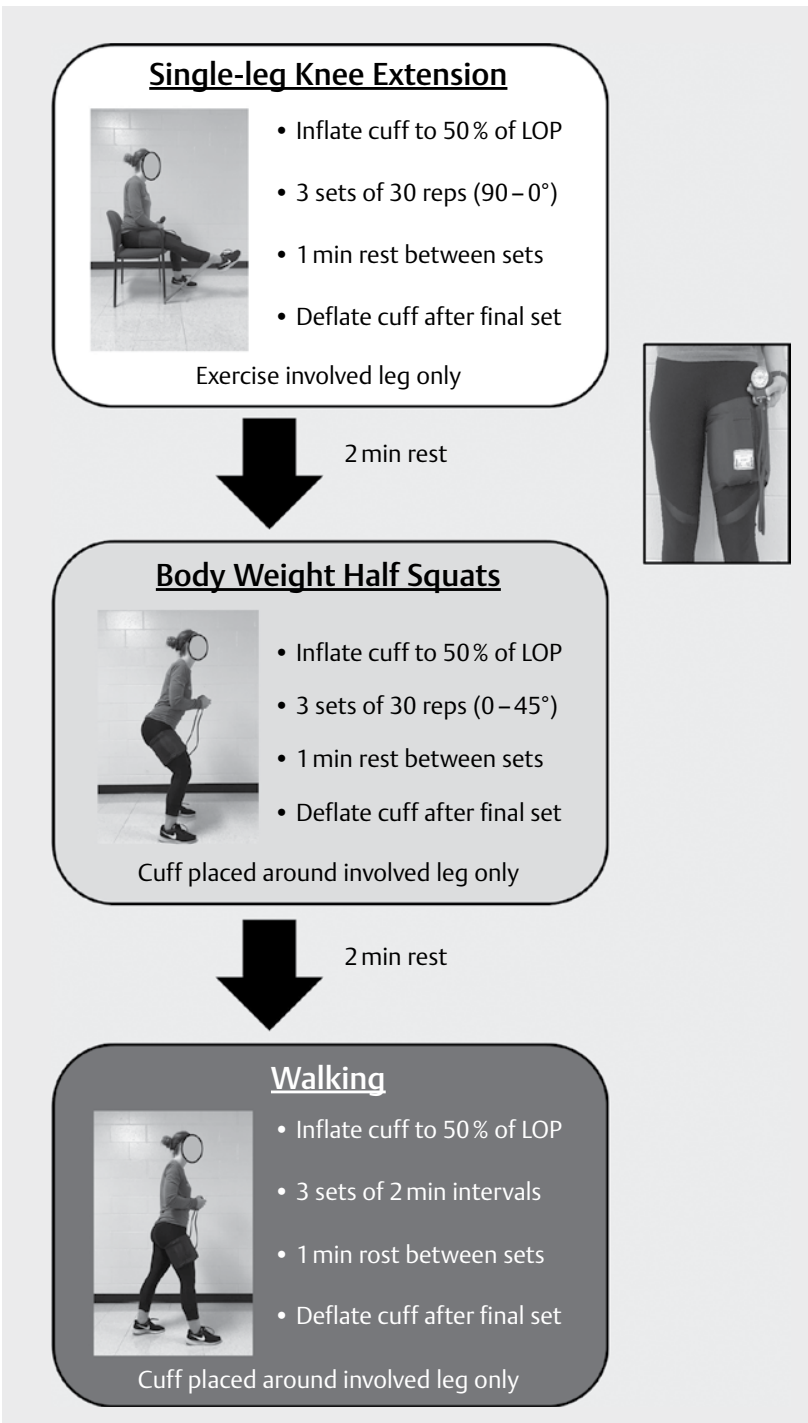

Fig. 1 Overview a home-based BFR exercise training session. Each training session consisted of a series of three exercises (single-leg knee extension, bodyweight half squats, walking) and took $\sim 25 \mathrm{~min}$ to complete. During each exercise, blood flow to the working muscles in the involved leg was restricted using a thigh cuff set to $50 \%$ of limb occlusion pressure. Inset image illustrates cuff placement over the involved leg. Participants performed 5 training sessions/wk for 4 wks. the superficial and deep aponeurosis [29]. Before and after training, measurements of muscle thickness were taken $5 \times /$ day on two separate days separated by at least 24 hrs. The average of the 10 measurements was used for analysis. Muscle thickness values in each leg were used to calculate symmetry index (described below).

Single-leg knee extensor strength was evaluated on a knee extension strength machine (Cybex, Life Fitness, Rosemont, IL, USA). Following a standardized warm-up, participants performed 10 repetitions at $\sim 80 \%$ of their maximum. Subsequently, $2.3-4.5 \mathrm{~kg}$ were added and 2 min of rest was provided. Participants were then given one attempt to successfully complete the 10-repetition knee extension task. If successfully completed with proper form, $2.3-4.5 \mathrm{~kg}$ were added and the participant attempted another 10 repetitions following a 2-min rest. This was repeated until the participant could no longer perform 10 repetitions with proper form. The investigator monitored the movement to ensure that full range of motion was achieved and that compensatory efforts were minimized. Weight and number of repetitions completed were recorded and 1 RM was estimated using Brzycki's equation [30]. Strength values were used to calculate symmetry index (described below).

\section{Symmetry index}

For the ACLR group, symmetry index was calculated as: SI = [(involved leg/uninvolved leg) * 100] [14]. Accordingly, a number less than $100 \%$ indicated that the involved leg had a lower value than the uninvolved leg. For the control group, the symmetry index was calculated as: $\mathrm{SI}=\left[\left(\right.\right.$ non-dominant leg/(dominant leg) $\left.{ }^{*} 100\right]$. The dominant leg was self-reported as the limb used to kick a ball [14]. A symmetry index less than $100 \%$ indicated that the non-dominant leg had a lower value than the dominant leg.

\section{Statistical analysis}

Independent t-tests were used to compare differences in demographic characteristics between the ACLR and uninjured control groups. Additionally, independent t-tests were used to assess differences in baseline rectus femoris and vastus lateralis thickness and knee extensor strength symmetry indices between the ACLR and uninjured control groups. A one-way repeated measures analysis of variance (ANOVA) was performed on muscle soreness values. Separate 2 (involved vs. uninvolved leg) $\times 2$ (baseline vs. posttraining) repeated measures ANOVA procedures were used to assess changes in rectus femoris and vastus lateralis thickness and knee extensor strength. Follow-up paired t-tests were used to test for simple main effects of leg and time. Paired t-tests were also used to assess differences in baseline and post-training symmetry indices for the ACLR group. Finally, independent t-tests were used to evaluate differences in symmetry indices between ACLR (post-training) and uninjured control groups (baseline). Alpha was set to 0.05 and data were presented as mean \pm SD.

\section{Results}

\section{Baseline}

Demographic characteristics between the ACLR and uninjured control groups did not differ (all $P>0.05$; $>$ Table 1). Participants in the ACLR group had surgery $5 \pm 2$ yrs ago with an autograft of semiten- 

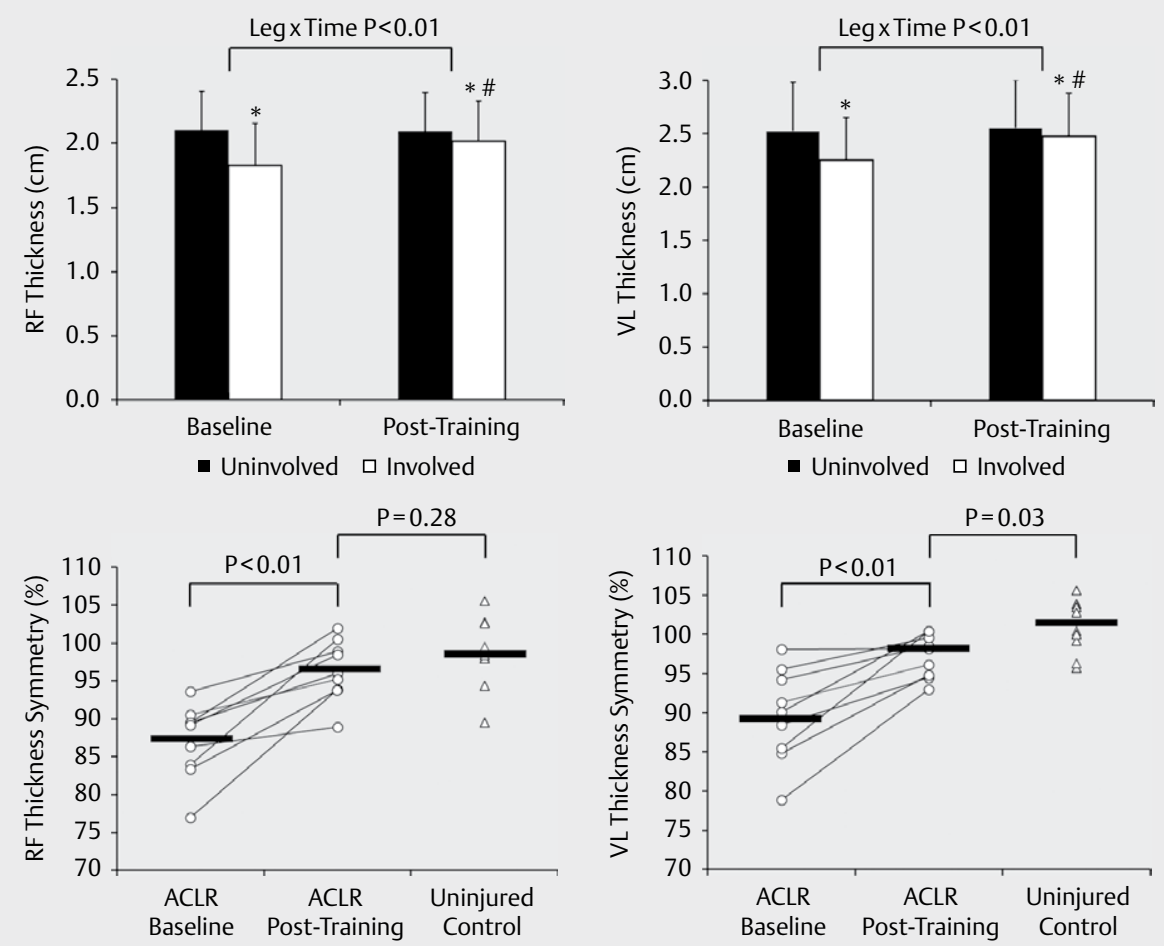

- Fig. 2 Alterations in rectus femoris (RF) and vastus lateralis (VL) thickness in the involved and uninvolved leg for the ACLR group (top panels). Muscle thickness symmetry in the ACLR and uninjured control groups (bottom panels). For the ACLR group, a number $<100 \%$ indicated uninvolved leg dominance. For the uninjured control group, a number $<100 \%$ indicated dominant leg dominance. Thin lines represent individual changes from baseline to post-training for the ACLR group and open triangles represent individual values for the uninjured control group. Horizontal thick lines represent mean values for $A C L R$ and uninjured control groups. Data are reported as mean $\pm S D$. ${ }^{*} P<0.05$ vs. uninvolved leg. ${ }^{\#} P<0.05$ vs. baseline.

dinosus muscle tendon $(n=3)$ or patellar tendon $(n=6)$. For these participants, rectus femoris and vastus lateralis thickness and knee extensor strength were reduced in the involved leg compared to the uninvolved leg (all $P<0.01$; $>$ Figs 2,3). As expected, symmetry values for rectus femoris ( $87 \pm 5 \%$ ) and vastus lateralis ( $90 \pm 6 \%$ ) thickness and knee extensor strength ( $88 \pm 4 \%$ ) were $\leq 90 \%$ and thus were in the moderate asymmetry range (29). Symmetry indices for the ACLR group were less than the uninjured control group (all $P<0.01$; $>$ Figs 2,3).

\section{BFR exercise}

Mean limb occlusion pressure was $182 \pm 28 \mathrm{mmHg}$. Accordingly, mean BFR training pressure was set at $91 \pm 14 \mathrm{mmHg}$. ACLR participants completed $98 \%$ of the total exercise sessions and there were no adverse events. Mean muscle soreness with the exercise training was $<1.0 \mathrm{~cm}$ and did not differ across training sessions $(P>0.05$; Fig. 4).

\section{Post-Training}

There were significant leg $x$ time interactions for rectus femoris and vastus lateralis thickness (both $\mathrm{P}<0.01$ ) indicating that the involved leg exhibited a greater change in quadriceps muscle thickness compared to the uninvolved leg ( $\triangleright$ Fig. 2). After training, rectus femoris and vastus lateralis thickness in the involved leg increased by $11 \pm 5 \%$ and $10 \pm 6 \%$ (both $P<0.01$ ), respectively, but did not change in the uninvolved $\operatorname{leg}(P=0.76, P=0.47)$. A small but signif- icant difference was present in rectus femoris and vastus lateralis thickness between the ACLR and uninvolved legs after training $(P=0.03, P=0.02)$. Post-training rectus femoris thickness symmetry in the ACLR group increased compared to baseline $(87 \pm 5$ to $96 \pm 4 \%, P<0.01)$ and did not differ from that for the uninjured control group ( $99 \pm 5 \%, P=0.28$; $\triangleright$ Fig. 2). Post-training symmetry for vastus lateralis thickness in the ACLR group increased compared to baseline ( $90 \pm 6$ to $97 \pm 3 \%, P<0.01$ ) but was lower than that for the uninjured control group (101 $\pm 3 \%, P=0.03$; - Fig. 2$)$.

There was also a significant leg $\times$ time interaction for knee extensor strength ( $P<0.01$, $>$ Fig. 2). After training, knee extensor strength increased by $20 \pm 14 \%$ in involved leg $(P<0.01)$ and did not change in the uninvolved leg $(P=0.09)$. Following training, knee extensor strength in the involved and uninvolved legs did not differ $(P=0.71)$. Post-training knee extensor strength symmetry in the ACLR group increased compared to baseline ( $88 \pm 4$ to $99 \pm 5 \%$, $\mathrm{P}<0.01)$ and did not differ from the uninjured control group $(99 \pm 5 \%, P=0.95 ;$ - Fig. 3$)$.

\section{Discussion}

\section{Main findings}

Participants in this study had an ACLR on average 5 yrs ago and, despite completing a post-operative rehabilitation program and being physically active, still had persistent quadriceps impairments and 


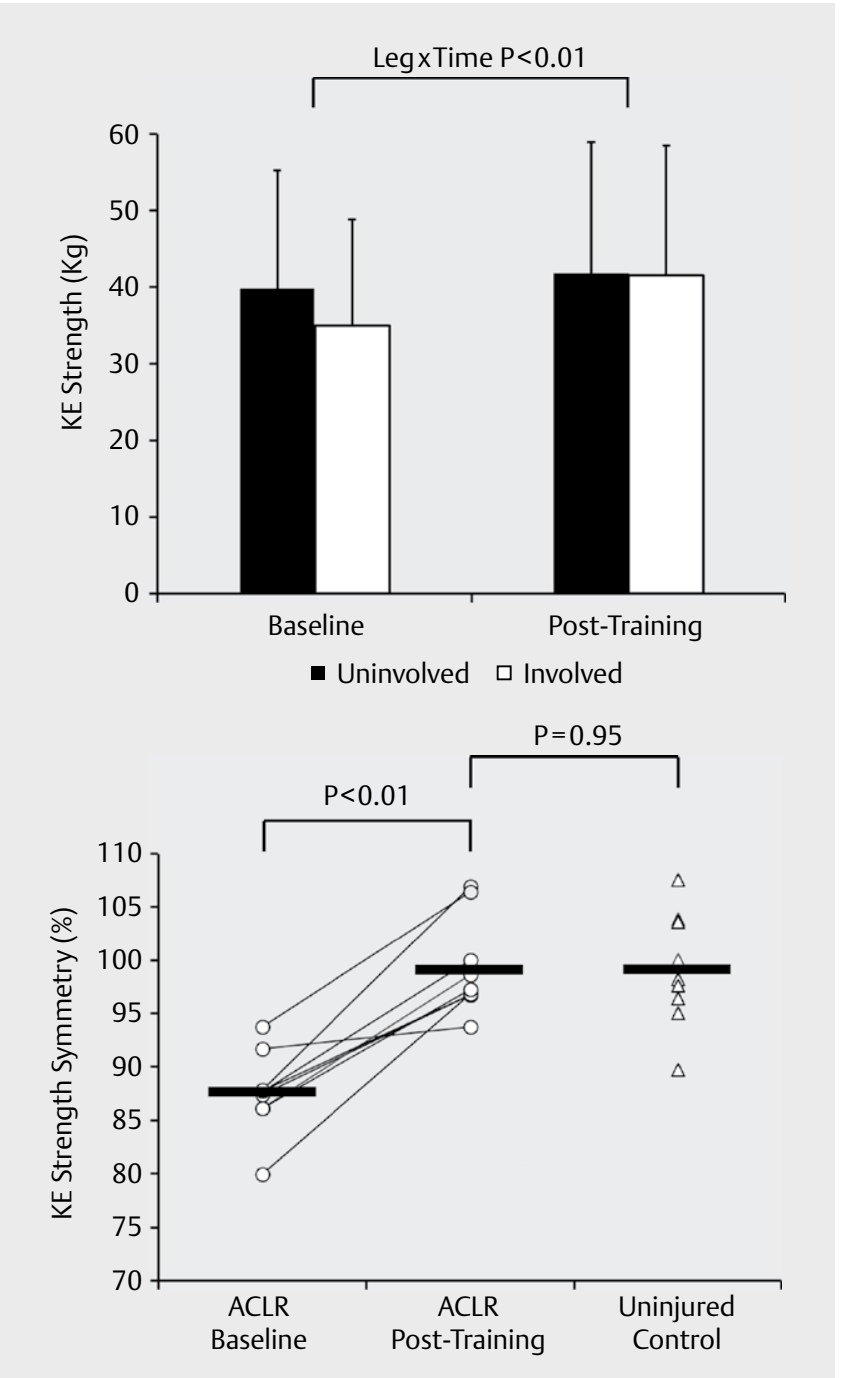

- Fig. 3 Changes in knee extensor 1RM strength in the involved and uninvolved leg for the ACLR group (top panel). Knee extensor 1RM strength symmetry in the ACLR and uninjured control groups (bottom panel). For the ACLR group, a number $<100 \%$ indicated uninvolved leg dominance. For the uninjured control group, a number $<100 \%$ indicated dominant leg dominance. Thin lines represent individual changes from baseline to post-training for the ACLR group and open triangles represent individual values for the uninjured control group. Horizontal thick lines represent mean values for ACLR and uninjured control groups. Data are reported as mean \pm SD.

${ }^{*} \mathrm{P}<0.05$ vs. uninvolved leg. ${ }^{\#} \mathrm{P}<0.05$ vs. baseline.

moderate asymmetry. Accordingly, we implemented a 4-wk, homebased BFR program consisting of bodyweight and walking exercises to increase quadriceps size and strength. As expected, BFR exercise increased rectus femoris and vastus lateralis thickness, as well as knee extensor strength to a greater extent in the involved leg compared to the uninvolved leg. Consequently, asymmetry in muscle thickness and knee extensor strength measures was reduced such that symmetry levels (96-99\%) were much closer to those of uninjured controls (99-101\%). These results support our hypotheses, shed light on the application of BFR exercise at home, and offer a promising approach for restoring quadriceps size and strength long after ACLR.

\section{Home-Based exercise with BFR}

A unique aspect of this study was the development of a homebased BFR exercise protocol. To minimize the need for expensive exercise equipment, we implemented simple exercises that only required the use of a thigh cuff and a resistance band ( $\sim 20$ USD). We also used moderate cuff pressures $(\sim 90 \mathrm{mmHg})$ normalized to individual limb occlusion pressure and educated participants on how to carefully monitor the pressure during a BFR exercise session. Using this approach, participants were able to perform BFR exercise without causing injury and tolerated the training as muscle soreness was very low. Thus, implementation of BFR exercise at home provided a program that was feasible, safe and inexpensive. Finally, it is important to point out that participants did receive specific instruction and careful monitoring from the research team. We also acknowledge that motivation to perform and tolerate BFR exercise could be different for individuals that are not participating in an organized study. Therefore, more work is needed to confirm the potential clinical applications of home-based BFR exercise.

\section{Quadriceps size and strength}

The moderate asymmetry present in the ACLR group before training was not surprising as several authors [13-15] have reported persistent quadriceps asymmetry following ACLR. After 4 wks of BFR exercise, rectus femoris and vastus lateralis thickness in the involved leg increased by 11 and $10 \%$, respectively. Increased quadriceps thickness is significant because knee extensor strength is more associated with quadriceps size rather than voluntary activation as individuals recovering from knee surgery pass the $1 \mathrm{yr}$ mark $[13,31,32]$. The home-based BFR exercise program also increased knee extensor strength by $20 \%(\sim 6.5 \mathrm{~kg})$. Together, these improvements in quadriceps muscle thickness and knee extensor 1RM strength are consistent with those for healthy adults performing BFR resistance [33] and aerobic [34] exercise where quadriceps volume and whole-leg 1 RM strength increased by $\sim 6 \%$ and $~ 12 \%$, respectively.

With increased muscle thickness and strength in the involved leg, there was a notable improvement in symmetry for rectus femoris (87-96\%) and vastus lateralis (90-97\%) thickness and knee extensor strength (88-99\%). Indeed, post-training symmetry values were much closer to those of uninjured controls. Most importantly, these participants that had an ACLR procedure were able to overcome presumably years of quadriceps weakness with just one month of targeted exercise training and achieve remarkable levels of knee extensor strength symmetry. These symmetry outcomes extend upon reports documenting the application of BFR exercise early after ACLR. For example, Ohta and colleagues [20] reported that BFR exercise implemented 3-16 weeks post-operatively increased knee extensor strength symmetry (65-77\%). Taken together, results from the current study along with previous reports $[19,20,35]$ clearly demonstrate that BFR exercise can offer a potent stimulus for improving quadriceps size and strength after ACLR. Moreover, implementation of exercise involving low loads to improve quadriceps size and strength in individuals that have a history of ACLR is noteworthy because high-load resistance exercise can be sometimes be contraindicated. 


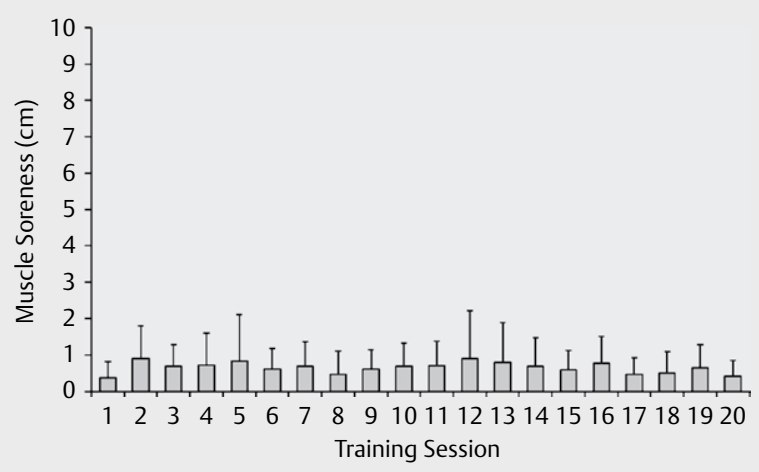

-Fig. 4 Perceived muscle soreness associated with the homebased BFR exercise program ( 0 - no soreness, 10 - severe soreness). Data are reported as mean \pm SD.

\section{Implications}

When patients with ACLR are eventually discharged from supervised rehabilitation, clinicians may consider incorporating BFR exercises into home-based maintenance programs. Specifically, this may allow patients to further increase their quadriceps size and strength, symmetry and/or physical function, because low load BFR resistance exercise is more effective than traditional low load resistance exercise [36]. An alternative possibility is that low load BFR resistance exercise may enable individuals to progress sooner towards high load resistance exercise [5], which elicits even greater strength gains. Additionally, individuals with ACLR that have persistent quadriceps impairments and require follow-up medical intervention may find BFR exercise practical, because it can be performed at home, facilitates strength gains quickly, and involves inexpensive equipment.

\section{Summary}

Implementation of BFR exercise several years after ACLR increased quadriceps muscle thickness and knee extensor strength and reduced asymmetry. Additionally, the home-based program consisting of bodyweight and walking BFR exercises was feasible, safe and inexpensive. We conclude that, for this ACLR cohort, home-based BFR exercise provided an effective intervention to improve quadriceps function long after ACLR.

\section{Acknowledgements}

The authors would like to sincerely thank the participants who took part in this study for their enthusiastic efforts. The authors also thank Kate Glodowski, Brenna Sellman, and Zach Bennett for their assistance with pilot data collection and Alicia Denherder and Thomas Bye for feedback on the manuscript. This research was supported by the Blue Cross Blue Shield of Michigan Foundation.

\section{Conflict of Interest}

\section{References}

[1] Konishi Y, Ikeda K, Nishino A, Sunaga M, Aihara Y, Fukubayashi T. Relationship between quadriceps femoris muscle volume and muscle torque after anterior cruciate ligament repair. Scand J Med Sci Sports 2007; 17: 656-661

[2] Marcon M, Ciritsis B, Laux C, Nanz D, Fischer MA, Andreisek G, Ulbrich E]. Quantitative and qualitative MR-imaging assessment of vastus medialis muscle volume loss in asymptomatic patients after anterior cruciate ligament reconstruction. J Magn Reson Imaging 2015; 42: 515-525

[3] Thomas AC, Wojtys EM, Brandon C, Palmieri-Smith RM. Muscle atrophy contributes to quadriceps weakness after anterior cruciate ligament reconstruction. J Sci Med Sport 2016; 19: 7-11

[4] Dejong SN, van Caspel DR, van Haeff MJ, Saris DB. Functional assessment and muscle strength before and after reconstruction of chronic anterior cruciate ligament lesions. Arthroscopy 2007; 23: 21-28, 28.e1-3

[5] Hughes L, Paton B, Rosenblatt B, Gissane C, Patterson SD. Blood flow restriction training in clinical musculoskeletal rehabilitation: A systematic review and meta-analysis. Br J Sports Med 2017; 51: 1003-1011

[6] Thomas AC, Villwock M, Wojtys EM, Palmieri-Smith RM. Lower extremity muscle strength after anterior cruciate ligament injury and reconstruction. J Athl Train 2013; 48: 610-620

[7] DiStasi SL, Logerstedt D, Gardinier ES, Snyder-Mackler L. Gait patterns differ between ACL-reconstructed athletes who pass return-to-sport criteria and those who fail. Am J Sports Med 2013; 41: 1310-1318

[8] Palmieri-Smith RM, Lepley LK. Quadriceps strength asymmetry after anterior cruciate ligament reconstruction alters knee joint biomechanics and functional performance at time of return to activity. Am J Sports Med 2015; 43: 1662-1669

[9] Schmitt LC, Paterno MV, Hewett TE. The impact of quadriceps femoris strength asymmetry on functional performance at return to sport following anterior cruciate ligament reconstruction. J Orthop Sports Phys Ther 2012; 42: 750-759

[10] Grindem H, Snyder-Mackler L, Moksnes H, Engebretsen L, Arna Risberg M. Simple decision rules reduce reinjury risk after anterior cruciate ligament reconstruction. Br J Sports Med 2016; 50: 804-808

[11] Oiestad BE, Holm I, Gunderson R, Myklebust G, Risberg MA. Quadriceps muscle weakness after anterior cruciate ligament reconstruction: A risk factor for knee osteoarthritis? Arthritis Care Res (Hoboken) 2010; 62: 1706-1714

[12] Tourville TW, Jarrell KM, Naud S, Slauterbeck JR, Johnson RJ, Beynnon BD. Relationship between isokinetic strength and tibiofemoral joint space width changes after anterior cruciate ligament reconstruction. Am J Sports Med 2014; 42: 302-311

[13] Krishnan C, Williams GN. Factors explaining chronic knee extensor strength deficits after $A C L$ reconstruction. J Orthop Res 2011; 29: 633-640

[14] Kuenze CM, Hertel J, Weltman A, Diduch D, Saliba SA, Hart JM. Persistent neuromuscular and corticomotor quadriceps asymmetry after anterior cruciate ligament reconstruction. J Athl Train 2015; 50: 303-312

[15] Longo UG, Rizzello G, Frnaceschi F, Campi S, Maffulli N, Denaro V. The architecture of the ipsilateral quadriceps two years after successful anterior cruciate ligament reconstruction with bone-patellar tendon-bone autograft. Knee 2014; 21: 721-725

[16] Garber CE, Blissmer B, Deschenes MR, Franklin BA, Lamonte M], Lee IM, Nieman DC, Swain DP. American College of Sports M. American College of Sports Medicine position stand. Quantity and quality of exercise for developing and maintaining cardiorespiratory, musculoskeletal, and neuromotor fitness in apparently healthy adults: Guidance for prescribing exercise. Med Sci Sports Exerc 2011; 43: 1334-1359 
[17] Barber-Westin S, Noyes FY. Blood-flow restriction training for lower extremity muscle weakness due to knee pathology: A systematic review. Sports Health 2019; 11: 69-83

[18] DePhillipo NN, Kennedy MI, Aman ZA, Bernhardson AS, O’Brien LT, LaPrade RF. The role of blood flow restriction therapy following knee surgery: Expert opinion. Arthroscopy 2018; 34: 2506-2510

[19] Takarada Y, Takazawa H, Ishii N. Applications of vascular occlusion diminish disuse atrophy of knee extensor muscles. Med Sci Sports Exerc 2000; 32: 2035-2039

[20] Ohta H, Kurosawa H, Ikeda H, Iwase Y, Satou N, Nakamura S. Low-load resistance muscular training with moderate restriction of blood flow after anterior cruciate ligament reconstruction. Acta Orthop Scand 2003; 74: 62-68

[21] Tennent DJ, Hylden CM, Johnson AE, Burns TC, Wilken JM, Owens JG. Blood flow restriction training after knee arthroscopy: A randomized controlled pilot study. Clin J Sport Med 2017; 27: 245-252

[22] Curran MT, Lepley LK, Palmieri-Smith RM. Continued improvements in quadriceps strength and biomechanical symmetry of the knee after postoperative anterior cruciate ligament reconstruction rehabilitation: Is it time to reconsider the 6-month return-to-activity criteria? Athl Train 2018; 53: 535-544

[23] Lewek M, Rudolph K, Axe M, Snyder-Mackler L. The effect of insufficient quadriceps strength on gait after anterior cruciate ligament reconstruction. Clin Biomech (Bristol, Avon) 2002; 17: 56-63

[24] Grant JA, Mohtadi NG, Maitland ME, Zernicke RF. Comparison of home versus physical therapy-supervised rehabilitation programs after anterior cruciate ligament reconstruction: A randomized clinical trial. Am J Sports Med 2005; 33: 1288-1297

[25] Harriss DJ, Macsween A, Atkinson G. Standards in sport and exercise science research: 2018 update. Int J Sports Med 2017; 38: 1126-1131

[26] Hunt JE, Stodart C, Ferguson RA. The influence of participant characteristics on the relationship between cuff pressure and level of blood flow restriction. Eur J Appl Physiol 2016; 116: 1421-1432

[27] Kilgas MA, McDaniel J, Stavres J, Pollock BS, Singer T], Elmer S]. Limb blood flow and tissue perfusion during exercise with blood flow restriction. Eur J Appl Physiol 2019; 119: 377-387
[28] Twist C, Eston R. The effects of exercise-induced muscle damage on maximal intensity intermittent exercise performance. Eur J Appl Physiol 2005; 94: 652-658

[29] Martin-Hernandez J, Marin P], Menendez H, Loenneke JP, Coelho-e-Silva M], Garcia-Lopez D, Herrero AJ. Changes in muscle architecture induced by low load blood flow restricted training. Acta Physiol Hung 2013; 100: 411-418

[30] Brzycki M. Strength testing: Predicting one-rep max from repetitions to fatigue. Journal of Physical Education Recreation \& Dance 1993; 64: 88-90

[31] Meier WA, Marcus RL, Dibble LE, Foreman KB, Peters CL, Mizner RL, LaStayo PC. The long-term contribution of muscle activation and muscle size to quadriceps weakness following total knee arthroplasty. J Geriatr Phys Ther 2009; 32: 35-38 13

[32] Petterson SC, Barrance P, Marmon AR, Handling T, Buchanan TS, Snyder-Mackler L. Time course of quad strength, area and activation after knee arthroplasty and strength training. Med Sci Sports Exerc 2011; 43: 225-231

[33] Abe T, Yasuda T, Midorikawa T, Sato Y, Kearns CF, Inoue K, Koizumi K, Ishii N. Skeletal muscle size and circulating IGF-1 are increased after two weeks of twice daily "KAATSU" resistance training. Int J KAATSU Training Res 2005; 1: 6-12

[34] Abe T, Kearns CF, Sato Y. Muscle size and strength are increased following walk training with restricted venous blood flow from the leg muscle, Kaatsu-walk training. J Appl Physiol (1985) 2006; 100: 1460-1466

[35] Lejkowski PM, Pajaczkowski JA. Utilization of Vascular Restriction Training in post-surgical knee rehabilitation: A case report and introduction to an under-reported training technique. J Can Chiropr Assoc 2011; 55: 280-287

[36] Loenneke JP, Wilson JM, Marin PJ, Zourdos MC, Bemben MG. Low intensity blood flow restriction training: A meta-analysis. Eur J Appl Physiol 2012; 112: 1849-1859 